\title{
Designing a DEMATEL Evaluation Model to Promote Health Tourism Development
}

\author{
Shan Shan Huang, Chien Chou Yu* \\ Department of Tourism Management, School of Economics and Management, Sanming University, Sanming, China
}

Email address:

chienchouyu@qq.com (Chien Chou Yu)

${ }^{*}$ Corresponding author

\section{To cite this article:}

Shan Shan Huang, Chien Chou Yu. Designing a DEMATEL Evaluation Model to Promote Health Tourism Development. Science Innovation. Vol. 9, No. 5, 2021, pp. 210-215. doi: 10.11648/j.si.20210905.16

Received: September 4, 2021; Accepted: October 21, 2021; Published: October 28, 2021

\begin{abstract}
Health tourism is an important policy for "implementing a healthy China strategy." China has a vast territory, and different regions have different levels of economic development and market sizes. The implementation of policies requires evaluation of resources and rankings in order to proceed in an orderly manner. Forest scenic spots are an important carrier for realizing health tourism. However, the current researches seldom discuss the quantitative evaluation of health resources in forest scenic spots. This article aims to propose a quantitative model to help local scenic spots to scientifically identify key resources to promote health tourism. This study sorted out the evaluation criteria through literature review; then designed an evaluation model using the decision-making experiment and evaluation laboratory (DEMATEL) method; finally, verified the operation processes of the model in a local scenic spot. The results show that the designed DEMATEL evaluation model can integrate expert opinions, calculate and convert data in the MS Excel software environment, and generate numerical and visual image information. These processes allow the scenic spot managers to identify key resources in an intuitive way, and find high-impact routs to plan corresponding constructions. This research provides a practical and easy-to-operate evaluation model, which can provide richer theoretical support and quantitative tools for promoting the development of healthy tourism in local scenic spots.
\end{abstract}

Keywords: Health Tourism, Decision-Making Trail and Evaluation Laboratory (DEMATEL) Method, Evaluation Model

\section{设计决策实验室评价模式推进地方景区康养旅游发展}

黄杉杉，俞建州"

三明学院旅游管理与服务教育系经济与管理学院, 三明, 中国

邮箱

chienchouyu@qq.com（俞建州）

摘要：康养旅游是“实施健康中国战略”的重要政策。中国幅员辽阔，不同区域经济发展水平和市场规模大小不一，政 策实现需要评价资源, 区分位次, 以利有序进行。森林景区是实现康养旅游的重要载体, 然而, 目前研究对于森林景 区康养资源的量化评价较少论及。本文旨在提出一个量化模型, 帮助地方景区科学识别关键资源推进康养旅游。本研 究通过文献查阅整理出评价指标; 使用决策试验和评估实验室（DEMATEL）方法设计评估模型；最后，在一个地方 景区验证了该模型的运行过程。结果表明，所设计的DEMATEL评价模型能够在MS Excel软件环境中整合专家意见并 计算和转换资料，生成数值和视觉图像信息。这些过程可以让森林景区管理者以直观的方式识别关键资源，找出具有 高影响力的路径来规划相应的建设。本研究提供了一个实用且易于操作的评价模型, 可为促进地方景区康养旅游发展 提供更丰富的理论支持和量化工具。 
关键词: 康养旅游, 决策实验室法 (DEMATEL), 评价模式

\section{1. 引言}

近年来，在“实施健康中国战略”的重大部署下，《关 于促进森林康养产业发展的意见》等相关政策相继出台, 整合旅游, 文化, 健康, 养老等要素, 形成“康养旅游”业 态, 在促进民众身心健康, 活络市场经济, 引领社会公卫 升级, 增加城市知名度等方面, 取得重大效果 [1]。

尽管目前我国康养旅游取得一定的成绩, 实践过程也 存在一些有待继续优化的问题。例如康养资源认知、开发 和利用处在初级探索阶段, 衍生同质化康养旅游产品服/ 务设计, 未充分结合特色资源体现核心价值, 不利长期发 展。其次, 康养旅游概念内涵和外延发展, 缺少成熟的理 论体系和实务典范; 又不同区域经济发展水平和市场规模 大小不一, 亟须通过评价, 发掘地方特色康养资源, 规划 发展路径, 保障政策实践效果 [2]。特别是我国幅员辽阔, 不同区域经济发展水平和市场规模大小不一, 政策实现需 要评价资源, 区分位次, 以利有序进行。

森林景区是实现康养旅游的重要载体, 然而, 目前森 林康养旅游的量化研究不多 [3]; 对于森林景区康养资源的 量化评价较少论及 [4]。本文旨在提出一个量化模型, 帮助 地方景区科学识别关键资源推进康养旅游。本研究通过文 献查阅整理出评价指标; 使用决策试验和评估实验室

(DEMATEL) 方法[5]设计评估模型；最后，在一个地方 景区验证了该模型的运行过程。结果表明, 所设计的 DEMATEL评价模型能够在MS Excel软件环境中整合专 家意见并计算和转换资料, 生成数值和视觉图像信息。这 些过程可以让森林景区管理者以直观的方式识别关键资 源, 找出具有高影响力的路径来规划相应的建设。本研究 提供了一个实用且易于操作的评价模型, 可为促进地方景 区康养旅游发展提供更丰富的理论支持和量化工具。

\section{2. 文献综述}

康养旅游起源于森林康养。森林康养旅游可以藉由旅 游康复疗养身心, 吸引广大层级旅客, 形成森林康养特色 旅游市场。20世纪初我国开始建立各种等级的森林公园, 设置森林康养场域; 近年来，在一系列的政策支持与推动 下, 森林康养汇集疗养、康复、观光、休闲、游㮩于一体 的特色旅游[6], 发展势头十分良好。

国际上, 德国和日本等国家走在森林康养发展前列。 19 世纪初, 慕尼黑大学Endres等提出林业自然效益—“福利” 理论, 开启森林应用概念。其后美国森林保健模式, 以日 本及森林浴模式相继发展 [7]; 进入21世纪后全球分级管理 体系建立，公民营机构采用收费与免费方式，为民众提供 多样化森林旅游康养场域 [8]。

近年在政府政策支持下, 我国各地方特色康养旅游目 的地发展同类型的康养旅游, 森林康养为其重要一环, 康 养资源成为热门研究议题。李济任等通过层次分析法构建 了森林康养旅游开发潜力评价指标体系, 并对森林康养旅
游开发潜力进行评价[9]。学者宋子健, 温泉平对蔡家川森 林康养区进行了康养资源研究, 根据 10 类森林康养产品 种类将森林康养基地主导功能汇总为 5 类, 最终确定了 46项指标, 以及各项指标的权重[10]。

当前研究文献采用层次分析法、问卷调查法、交互式 过滤产生法、频度统计法、理论分析法与专家咨询法等方 法, 梳理评价指标, 区分森林康养资源项目的重要顺序; 然而当前文献以定性研究较多, 实证研究缺乏, 量化分析 的案例极少 [11]。此外这些研究并未运用评价指标发展量 化模式, 探讨分析特色资源与推进康养旅游路径问题 [12]; 因此, 本研究在现有定性文献研究的基础上, 采用定量方 法, 设计康养旅游资源评价模式, 协助推进康养旅游发展。

\section{3. 设计评价模式}

本章设计评价模式; 为利说明, 首先介绍设计模式所 采用之决策实验室方法的背景与运算步骤; 其次说明评价 模式。

\section{1. 决策实验室方法背景与运算步骤}

决策实验室法是由日内瓦的 Battelle 协会在1971年 提出的决策方法, 用来协助处理与人类生活有关的复杂问 题[13]。此方法能够量测问题有关变量的相互影响关系, 分析每一个变量在问题系统的中心度以及因果特性, 并且 绘制影响关联图来展现问题系统中个变量的影响程度与 方向 [14]; 因此广受学术与实务领域用来处理决策有关评 价、排序与选择 $[15,16]$ 。

决策实验室法收集领域专家意见, 按以下五个步骤运 算资料转换信息 $[16]$ 。

步骤1. 建立影响关系矩阵

专家问卷资料进行清理并确定有效后，以直线平均方 式将资料整合为影响关系矩阵 $\mathrm{D}$; 详如下列公式(1)。

$$
D=\left[\mathrm{d}_{\mathrm{ij}}\right]_{\mathrm{m} \times \mathrm{m}}
$$

上式中, $\mathrm{d}_{\mathrm{ij}}=\left(\sum_{\mathrm{K}=1}^{\mathrm{P}} \mathrm{o}_{\mathrm{ij}}^{\mathrm{k}}\right) / P, \mathrm{o}_{\mathrm{ij}}^{\mathrm{k}}$ 为第 $\mathrm{k}$ 位受测者认为 因素 $i$ 对于因素 $j$ 的影响程度; $P$ 为总受测人数; $m$ 为评价因 素的总数。

步骤2. 建立正规化影响关系矩阵

以矩阵 $D$ 各行向量与列向量加总之极大值矩阵中各元 素进行正规化, 得到正规化影响关系矩阵 $N$; 详如下列公 式(2)与公式(3)。

$$
N=\left[\mathrm{n}_{\mathrm{ij}}\right]_{\mathrm{m} \times \mathrm{m}}=\mathrm{D} / \mathrm{u}
$$

上式中，

$$
\mathrm{u}=\max \left(\sum_{\mathrm{i}=1}^{\mathrm{m}} \mathrm{n}_{\mathrm{ij}}, \sum_{\mathrm{j}=1}^{\mathrm{m}} n_{\mathrm{ij}}\right)
$$

步骤3. 建立总影响关系矩阵 
矩阵 $\mathrm{N}$ 进行马可夫随机运算, 建立总影响关系矩阵 $\mathrm{T}$; 详如下列公式(4)。

$$
T=\left[\mathrm{t}_{\mathrm{ij}}\right]_{\mathrm{m} \times \mathrm{m}}=\sum_{q=1}^{\infty} N^{q}=N(I-N)^{-1}
$$

上式中, 设 $q \rightarrow \infty, \lim N^{q}=0$; I为单位矩阵。

步骤4. 制作影响关连图

首先加总矩阵T中各行向量与列向量后, 产出新的向 量 $\mathrm{h}$ 与向量 $\mathrm{v}$; 计算方式详如下列公式(5)与公式6)。

$$
h=\left[\mathrm{h}_{\mathrm{r}}\right]_{\mathrm{m} \times 1}
$$

上式中, $h r=h_{r}=\sum_{j=1}^{m} t_{i j}, r=1,2, \ldots, m$.

$$
v=\left[\mathrm{v}_{\mathrm{c}}\right]_{1 \times \mathrm{m}}
$$

上式中, $v_{c}=\sum_{\mathrm{j}=1}^{\mathrm{m}} \mathrm{t}_{\mathrm{ij}}, \mathrm{c}=1,2, \ldots, \mathrm{m}$.

其次, 将向量 $h$ 加上向量 $v$ 的逆向量 $v^{-1}$, 得到中心度向 量 $c l$; 将向量 $h$ 减去向量 $v^{-1}$, 得到原因度向量 $c r$ 。 ; 详如下 列公式(7)与公式(8),

$$
\begin{aligned}
& c l=\left[\mathrm{cl}_{\mathrm{q}}\right]_{\mathrm{m} \times 1}=h+v^{-1} \\
& c r=\left[\mathrm{cr}_{\mathrm{q}}\right]_{\mathrm{m} \times 1}=\mathrm{h}-r v^{-1}
\end{aligned}
$$

上式中, 以上中心度向量 $c l$ 显示系统中各项因素对于 达成系统目标的直接影响和间接影响程度的总合。原因度
向量 $c r$ 显示系统中各项因素对于达成系统整体目标的性 质和程度; 当 $\mathrm{cr}_{\mathrm{q}}>0$ 时, 所对应的因素是影响系统绩效 的“因”，在达成系统目标的过程中，对系统中其它因素会 有一定程度的影响力; 当 $\mathrm{cr}_{\mathrm{q}}<0$ 时, 所对应的因素是影响 系统绩效的“果”，在达成系统目标的过程系统中，会受到 其它因素一钉程度的影响。

步骤4. 建立影响关係图

最终, 运用中心度向量 $c l$ 和原因度向量 $c r$, 在二维直 角坐标平面中绘制出影响关係图, 协助决策者透过可视化 的信息，有效掌握问题的关键。

步骤5. 计算影响关係权重

取矩阵 $\mathrm{T}$ 的对角线数据进行正规化运算, 得到影响权 重向量w; 详如下列公式(9)。

$$
w=\left[\mathrm{w}_{q}\right]_{\mathrm{m} \times 1}
$$

上式中, $w_{q}=\mathrm{t}_{\mathrm{ij}} / \sum_{i=j=1}^{m} \mathrm{t}_{\mathrm{ij}} ; \mathrm{t}_{\mathrm{ij}} \in T ; q=1,2 . \cdots m$. 影响权重向量 $w$ 显示了系统中各项因素对于达成系统 目标的相对重要性。

\section{2. 设计评价模式}

根据上述决策实验室方法所设计之地方森林康养资 源评价模式如下图1所示。

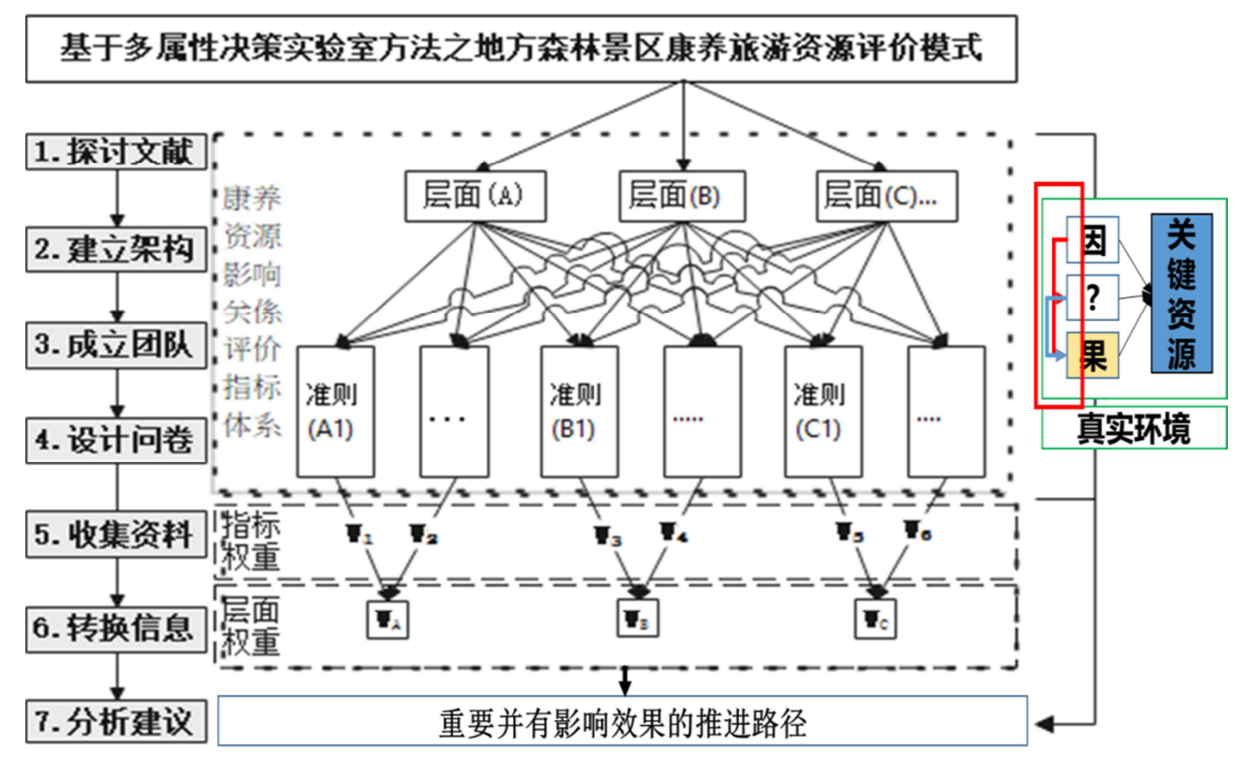

图1 评价模式。

图1所示评价模式通过探讨文献到分析建议等 7 个阶 段, 将康养旅游资源分解为可评价的层级式指标架构; 据 以设计问卷收集资料; 最终取得因果关係与重要位次二个 层面的数据与图像信息, 进行景区康养资源分析, 区分资 源位次, 铺垫相应路径建议。

\section{4. 模式验证}

本章依托地方景区实际验证所提出之评价模式的操 作性与实用性。

\section{1. 实证背景}

三明市根据《国家林业和草原局办公室 民政部办公 厅国家卫生健康委员会办公厅国家中医药管理局办公室 关于开展国家森林康养基地建设工作的通知》要求, 经省 级推荐、专家评审等程序, 建设三明市三元区格氏栲森林 康养基地。本文通过评价模式理清三明市三元格氏栲森林 康养基地存在的资源, 提出针对性的路径协助推动三明市 特色森林康养旅游发展。 


\section{2. 实证指标}

实证首先根据实证景区特性，依循第二章所探讨之康养旅游领域文献，建立层级式评价指标，包括 3 个层面共 8 项 准则; 详如下表1。

表1 评价指标说明表。

\begin{tabular}{llll}
\hline 目标 & 层面 & 属性 & 属性说明 \\
\hline & 自然资源 $(\mathrm{A})$ & 林草康养资源 $(\mathrm{A} 1)$ & 区域内植物的种类数量, 特有物种 \\
& & 农业康养资源 $(\mathrm{A} 2)$ & 绿色健康种植, 康养农产品, 药用价值农产品, 特色性康养农产品 \\
& & 文化环境 $(\mathrm{B} 1)$ & 传统孝文化与养生文化, 康养教育与才才培养, 康养会展与节事, 居民友好度 \\
地方森林 & 环境 $(\mathrm{B})$ & 人居环境 $(\mathrm{B} 2)$ & 环境质量, 居住条件, 基础设施, 交通出行 \\
景区康养 & & 医疗设施 $(\mathrm{C} 1)$ & 景区矢务室数量, 执业医生数, 医疗范围 \\
旅游资源 & & 养老设施 $\left(\mathrm{C}_{2}\right)$ & 养老服务机构的数量和等级, 老年友好设施覆盖率 \\
评价 & & 休闲设施 $\left(\mathrm{C}_{3}\right)$ & 康养设施规模及分布, 公共休闲空间的规模及分布, 公共体育休闲设施的规模及分布 \\
& 设施 $(\mathrm{C})$ & 康养品牌 $(\mathrm{C} 4)$ & 获康养相关奖项称号的数量, 康养品牌知名度 \\
& & &
\end{tabular}

\section{3. 实证资料与信息}

依据表1设计问卷, 邀请10位专业领域学者填答问卷均回收清理有效后, 按公式(1)计算汇整为直接影响矩阵 $\boldsymbol{A}$; 如 下表2所示。

表2 直接影响矩阵 $A$ 。

\begin{tabular}{|c|c|c|c|c|c|c|c|c|}
\hline $\mathbf{A}$ & $\begin{array}{l}\text { 林草康养 } \\
\text { 资源 }\left(\mathbf{A}_{11}\right)\end{array}$ & $\begin{array}{l}\text { 农业康养资源 } \\
\left(\mathbf{A}_{12}\right)\end{array}$ & 文化环境 $\left(\mathbf{B}_{21}\right)$ & 人居环境( $\left.\mathbf{B}_{22}\right)$ & 医疗设施 $\left(\mathrm{C}_{31}\right)$ & 养老设施 $\left(\mathrm{C}_{32}\right)$ & 休闲设施 $\left(\mathrm{C}_{33}\right)$ & 康养品牌 $\left(\mathbf{C}_{34}\right)$ \\
\hline 林草康养资源 $\left(\mathrm{A}_{11}\right)$ & 0.000 & 3.100 & 2.300 & 3.100 & 1.400 & 2.200 & 2.200 & 2.100 \\
\hline 农业康养资源 $\left(\mathrm{A}_{12}\right)$ & 3.100 & 0.000 & 2.100 & 2.700 & 1.800 & 2.000 & 2.000 & 2.400 \\
\hline 文化环境（B ${ }_{21}$ ) & 2.000 & 1.900 & 0.000 & 3.000 & 1.800 & 2.200 & 2.600 & 3.100 \\
\hline 人居环境(B 22$)$ & 2.700 & 2.500 & 3.400 & 0.000 & 3.100 & 3.100 & 3.100 & 3.400 \\
\hline 医疗设施（ $\mathrm{C}_{31} ）$ & 1.500 & 1.000 & 2.200 & 3.000 & 0.000 & 3.400 & 2.100 & 3.500 \\
\hline 养老设施（ $\mathrm{C}_{32}$ ） & 2.100 & 1.900 & 2.400 & 3.000 & 3.100 & 0.000 & 3.000 & 3.400 \\
\hline 康养品牌（ $\mathrm{C}_{34} ）$ & 2.900 & 2.700 & 3.200 & 3.200 & 3.000 & 3.400 & 2.800 & 0.000 \\
\hline
\end{tabular}

其次依据表2, 按公式(2)到(4), 进行数据正规化运算后建立总影响关系矩阵T, 如下表3所示。

表3 总影响关系矩阵T。

\begin{tabular}{|c|c|c|c|c|c|c|c|c|}
\hline $\mathbf{T}$ & $\begin{array}{l}\text { 林草康养资源 } \\
\left(\mathbf{A}_{11}\right)\end{array}$ & $\begin{array}{l}\text { 农业康养资源 } \\
\left(\mathbf{A}_{12}\right)\end{array}$ & $\begin{array}{l}\text { 文化环境 } \\
\left(\mathbf{B}_{21}\right)\end{array}$ & 人居环境( $\left.\mathbf{B}_{22}\right)$ & $\begin{array}{c}\text { 医疗设施 } \\
\left(\mathbf{C}_{31}\right)\end{array}$ & $\begin{array}{c}\text { 养老设施 } \\
\left(\mathbf{C}_{32}\right)\end{array}$ & $\begin{array}{c}\text { 休闲设施 } \\
\left(\mathbf{C}_{33}\right)\end{array}$ & $\begin{array}{c}\text { 康养品牌 } \\
\left(\mathbf{C}_{34}\right)\end{array}$ \\
\hline 林草康养资源（ $\mathrm{A}_{11}$ ） & 0.545 & 0.630 & 0.700 & 0.799 & 0.605 & 0.715 & 0.681 & 0.759 \\
\hline 农业康养资源（ $\mathrm{A}_{12} ）$ & 0.663 & 0.494 & 0.682 & 0.773 & 0.611 & 0.697 & 0.663 & 0.758 \\
\hline 文化环境（B ${ }_{21}$ ) & 0.642 & 0.595 & 0.618 & 0.811 & 0.635 & 0.732 & 0.710 & 0.813 \\
\hline 人居环境(B 22$)$ & 0.798 & 0.736 & 0.899 & 0.850 & 0.813 & 0.915 & 0.869 & 0.986 \\
\hline 医疗设施（ $\mathrm{C}_{31} ）$ & 0.630 & 0.566 & 0.722 & 0.823 & 0.570 & 0.790 & 0.703 & 0.842 \\
\hline 养老设施（ $\mathrm{C}_{32}$ ） & 0.711 & 0.654 & 0.792 & 0.894 & 0.751 & 0.716 & 0.797 & 0.908 \\
\hline 休闲设施（ $\mathrm{C}_{33}$ ） & 0.674 & 0.617 & 0.758 & 0.843 & 0.668 & 0.783 & 0.629 & 0.837 \\
\hline 康养品牌（ $\mathrm{C}_{34} ）$ & 0.802 & 0.740 & 0.888 & 0.976 & 0.806 & 0.921 & 0.854 & 0.843 \\
\hline
\end{tabular}

接续依据表3, 按公式(5)到(8), 及公式(9)计算各项评价指标的总影响程度、总被影响程度、中心度、原因度和权 重数据; 计算结果如下表4所示。

表4 评价指标相关信息汇总表。

\begin{tabular}{lllll}
\hline \multirow{2}{*}{ 评价指标 } & 相关信息 & & \\
\cline { 2 - 5 } & 总影响程度 & 总被影响程度 & 中心度 & 原因度 \\
\hline 林草康养资源 $\left(\mathrm{A}_{11}\right)$ & 5.434 & 5.465 & 10.899 & -0.031 \\
农业康养资源 $\left(\mathrm{A}_{12}\right)$ & 5.342 & 5.032 & 10.374 & 0.104 \\
文化环境 $\left(\mathrm{B}_{21}\right)$ & 5.556 & 6.059 & 11.615 & 0.09 \\
人居环境 $\left(\mathrm{B}_{22}\right)$ & 6.866 & 6.770 & 13.636 & -0.502 \\
医疗设施 $\left(\mathrm{C}_{31}\right)$ & 5.647 & 5.460 & 11.106 & 0.096 \\
养老设施 $\left(\mathrm{C}_{32}\right)$ & 6.223 & 6.270 & 12.493 & 0.187 \\
休闲设施 $\left(\mathrm{C}_{33}\right)$ & 5.809 & 5.906 & 11.715 & -0.047 \\
康养品牌 $\left(\mathrm{C}_{34}\right)$ & 6.831 & 6.747 & 13.578 & -0.097 \\
\hline
\end{tabular}


表4评价数据显示，景区各项康养资源对于旅游康养 的运用上, 有其原因度之下的主动与被动特性; 以及权重 下的主、次要优先顺序; 换言之, 评价模式所产生之原因 度与权重可以协助景区管理者透过数据分析筹划以最有
影响效果的方式，运用景区资源推进康养旅游。根依据表 4 , 按前章三、(二)、步骤4. 所述程序, 可以运用数据资料 绘制影响关联图(如下图3), 进一步展现康养资源运用的视 觉化决策信息。

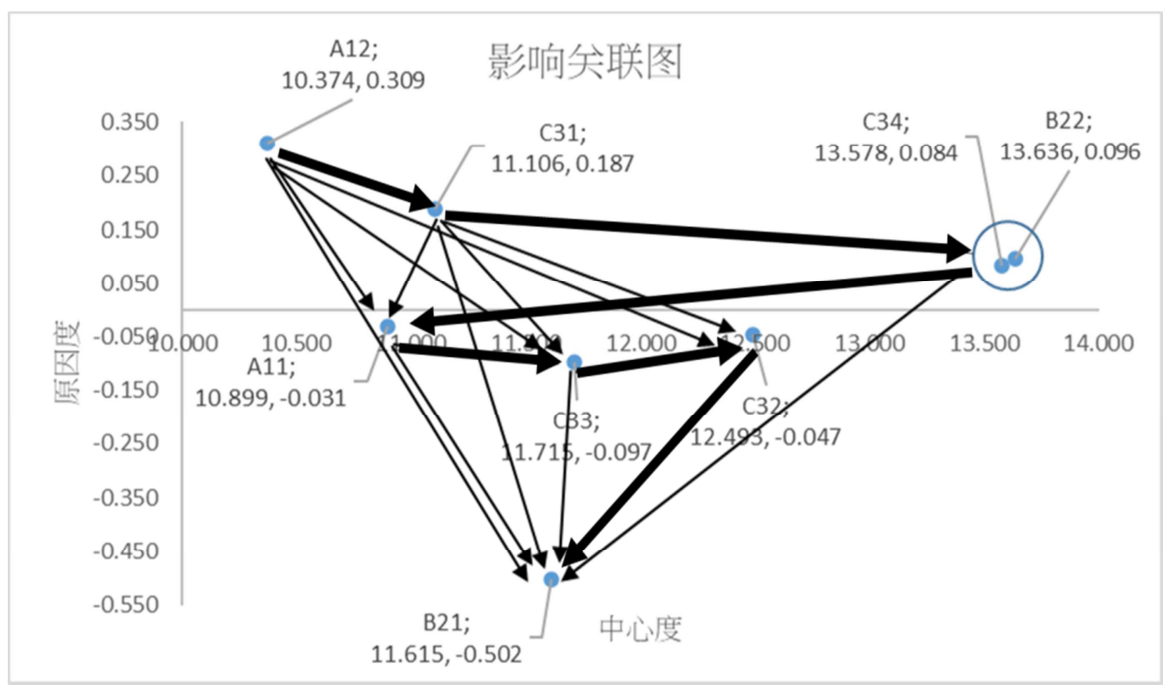

图3 影响关系图。

图3显示, 专家小组成员平均认为, 三元区格氏栲森 林景区运用康养资源推进康养旅游发展的首要考量为; 农 业康养资源 $\left(\mathrm{A}_{12}\right)$ 、医疗设施 $\left(\mathrm{C}_{31}\right)$ 、康养品牌 $\left(\mathrm{C}_{34}\right)$ 与人居环境 $\left(\mathrm{B}_{22}\right)$; 其后依序为林草康养资源 $\left(\mathrm{A}_{11}\right)$ 、休 闲设施 $\left(\mathrm{C}_{33}\right)$ 、养老设施 $\left(\mathrm{C}_{32}\right)$ 、文化环境 $\left(\mathrm{B}_{21}\right)$ 。因 为循此依顺序会有最大的相互推进影响效果。

\section{5. 结论发现与建议}

为推进地方景区康养旅游发展, 本文运用发展应用成 熟的决策实验室方法, 设计景区康养资源评价模式, 并实 际验证模式的操作性与实用性; 研究过程与结果表明。

\section{1. 运用科学方法设计评价模式有助于地方景区康养旅 游发展}

本研究所获各项信息显示, 运用多属性决策方法设计 评价模式可以展现景区各项康养资源的重要性、中心度、 与因果度(详如表4); 协助管理者评价有影响力的路径(详 如图3), 推进康养旅游。

\section{2. 设计地方景区康养旅游资源评价模式要考虑简单实 用}

研究过程发现地方景区多数人力层级属于技术业务 人员; 因此所设计评价之模式均运用办公室软件 EXCEL 内键函数进行所有运算与绘图; 此项容易操作使用的设计 考量, 对于景区人员了解评价模式, 迅速接受实际使用起 到相当大的作用。

本研究运用科学方法设计简易实用评价模式对于推 进康养旅游有其正向效果; 惟所设计之评价模式以单一景 区取得验证数据会存在小样本下的偏误; 建议后续研究者
可以参考本研究流程与评价模式, 针对其他不同类型景区, 调整评价指标与专家才队成员, 收集更多评价信息, 全面 推进国家景区康养旅游发展。

\section{致谢}

本文作者感谢黄三麟博士在研究过程的指导。感谢三 明学院KD190013项目经费支持。

\section{参考文献}

[1] 何䒭,彭菲,杜洁,沈山,崔永伟. 康养蓝皮书:中国康养产业发 展报告 (2019) [R]. 社会科学文献出版社,2019.

[2] 何莽,彭菲,杜洁,沈山,崔永伟. 康养蓝皮书:中国康养产业发 展报告（2020）[R]. 社会科学文献出版社,2021.

[3] Gülçin B., Esin M., Elif K. Health tourism strategy selection via SWOT analysis and integrated hesitant fuzzy linguistic AHP-MABAC approach [J]. Socio-Economic Planning Sciences, Volume 74, 2021. https://doi.org/10.1016/j.seps.2020.100929.

[4] 邵望,王庆生. 国内外康养旅游研究进展与前瞻[J].可持续发 展 ， 2020， 10(5)： 713-723. https://doi.org/10.12677/SD.2020.105088.

[5] Gabus A, Fontela E. World Problems an invitation to Additional thought within the framework of DEMATEL [J]. Switzerland Geneva: Battelle Geneva Research Center, 1972.

[6] 陈星.三明市森林康养旅游发展现状及对策分析 [J].海峡科 学, 2020,(03):72-75. 
[7] Lucie P, Lucie S. Enironment asa Key Factor of Health and Wel-Beiing Tourism Destinations in Five European Countries [J]. IBIMA Business Revien. VoL. 7. 2019.

[8] 周功梅, 宋瑞, 刘倩倩. 国内外康养旅游研究评述与展望 [J/OL]. 资源开发与市场, 2020, 1-19.

[9] 李济任,许东.森林康养旅游评价指标体系构建研究 [J]. 林业 经济, 2018,40(03):28-34.

[10] 宋子健,温全平.森林康养资源评价指标体系构建及评价 — 以蔡家川森林康养区为例 [J]. 林业科技情 报,2020,52(01):38-43.

[11] 肖光明,吴楚材.我国森林浴的旅游开发利用研究 [J].北京第 二外国语学院学报,2008(03):70-74.

[12] 束怡,楼毅,张宏亮,汪涵.我国森林康养产业发展现状及路径 探析—基于典型地区研究 [J]. 世界林业研 究,2019,32(04):51-56.
[13] 王伟明,徐海燕,朱建军.复杂网络视角下的大规模群体 DEMATEL 决策方法 [J]. 系统工程理论与实 践,2021,41(01):200-212.

[14] Chang, D. S., Wu W. D. Impact of the COVID-19 Pandemic on the Tourism Industry: Applying TRIZ and DEMATEL to Construct a Decision-Making Model. [J]. Sustainability. [J]. 13 (14), 2021. https://doi.org/10.3390/su13147610.

[15] 宋娜, 周旭瑶, 唐亦博, 潘越. 基于 DEMATEL- ISM一 MICMAC法的康养旅 游资源评价指标体系研究[J].生态经 济,2020,5,128-1390.

[16] Yu, C. C., Li, X., Lu, H. A Novel Procedure to Pursue Aspired Procurement Negotiation Outcomes Using a Combined MADM Model [J]. Mathematical Problems in Engineering Volume 2021, Article ID 8833250, 17 pages. http://dx.doi.org/10.1155/2021/8833250. 